\title{
Computational Study on the Nanotubes Formation between Olsalazine and $\beta$-Cyclodextrin
}

\author{
Abdulilah Dawoud Bani-Yaseen \\ Department of Chemistry and Earth Sciences, College of Arts and Sciences, Qatar University \\ P.O. Box 2713, Doha, State of Qatar \\ abdulilah.baniyaseen@qu.edu.qa
}

\section{Extended Abstract}

Interests in cyclodextrin-derived nano-assembled supramolecular systems have recently grown notably [1,2]. Cyclodextrins are a family of oligosaccharides that possess very characteristic features of conical shape with hydrophobic and hydrophilic interiors and exteriors, respectively. As a host molecule, cyclodextrin (CD) can encapsulate various types of molecules inside its cavity as host-guest supramolecular interaction that is in turn stabilized by non-covalent interactions [3-5]. Under specific conditions, cyclodextrins can self-aggregate to form nanoarchitectures, such as nanotubes and nanorods. However, guest-induced nanotubular architecture can be assembled with gust molecules of suitable size, such as olsalazine drug. The objective of this work is to computationally investigate the formation and the corresponding molecular properties of olsalazine- $\beta$-CD nanotubes using the semi-empirical method (SQM) PM7. Different guest:host ratios of inclusion complexes that can lead to the formation of the olsalazine- $\beta$-CD nanotubes were examined, namely $1: 2$ and 2:2. Furthermore, the side of penetration of the guest molecule is another factor that is considered herein, where head and tail inclusions correspond to the penetration inside the cavity of $\beta$-CD through the wide and narrow rims of $\beta$-CD, respectively. On the other hand, the head-to-head, tail-to-tail, head-to-tail aggregations of $\beta$-CD nanotubes are considered. The binding energy $\left(\mathrm{E}_{\mathrm{bind}}\right)$ of the nano suprastructure was calculated using the following formula:

$$
E_{\text {binding }}=\left(E_{\text {opt }}\right)_{\text {nanotube }}-m \cdot\left(E_{\text {opt }}\right)_{\text {olsa }}-n \cdot\left(E_{\text {opt }}\right)_{\beta-C D}
$$

where, $\mathrm{E}_{\mathrm{opt}}$ is the total energy of the optimized geometry of the free molecule, $m$ and $n$ correspond to the number of molecules of Olsalazine and $\beta-\mathrm{CD}$ associated in the formation of olsalazine- $\beta-C D \_n a n o t u b e s$, respectively.

Based on the values obtained for $E_{b i n d}$, Olsalazine can induce the formation of $\beta$-CD nanotube through the tail-to-tail inclusion, where one molecule of Olsalazine is included through the narrow rims of two $\beta$-CD molecules, whereas the aggregation can further grow through the head-to-head interaction of the $\beta$-CD molecules. It is demonstrated that the inclusion process is mainly stabilized by intermolecular noncovalent bonding, namely hydrogen bonds, where an average H-bond of approximately $1.75 \AA$ was estimated. The computational studies were conducted for the olsalazine- $\beta$-CD nanotubes within the ratios 1:2-5:10, which in turn revealed nanotubes with length and width of $\sim 8.00 .6 \mathrm{~nm}$, respectively, for the 5:10 ratio. Further results considering various types of parameters, such as thermodynamic parameters, associated with the formation of the olsalazine- $\beta$-CD nanotubes will be presented and discussed. The findings of this work considering the factors and mechanisms influencing the induced formation of $\beta-\mathrm{CD}$ nanotubes are insightful as such studies van enhance our understanding of this important phenomenon with more potential applications such as drug delivery

\section{References}

[1] N. Rajendiran and R. K. Sankaranarayanan, "Azo dye/cyclodextrin: New findings of identical nanorods through 2:2 inclusion complexes," Carbohydr. Polym., vol. 106, no. 1, pp. 422-431, 2014.

[2] F. B. De Sousa, A. C. Lima, Â. M. L. Denadai, C. P. a. Anconi, W. B. De Almeida, W. T. G. Novato, H. F. Dos Santos, C. L. Drum, R. Langer, and R. D. Sinisterra, "Superstructure based on $\beta$-CD self-assembly induced by a small guest molecule," Phys. Chem. Chem. Phys., vol. 14, pp. 1934-1944, 2012. 
[3] G. Crini, "Review: a history of cyclodextrins.," Chem. Rev., vol. 114, no. 21, pp. 10940-75, 2014.

[4] M. Ceborska, K. Szwed, M. Asztemborska, M. Wszelaka-Rylik, E. Kicińska, and K. Suwińska, "Study of $\beta$ cyclodextrin inclusion complexes with volatile molecules geraniol and $\alpha$-terpineol enantiomers in solid state and in solution," Chem. Phys. Lett., vol. 641, pp. 44-50, 2015.

[5] A. D. Bani-Yaseen, "Synchronous spectrofluorimetric study of the supramolecular host-guest interaction of $\beta$ cyclodextrin with propranolol: A comparative study.," Spectrochim. Acta. A. Mol. Biomol. Spectrosc., vol. 148, pp. 93-8, 2015. 